\title{
Quantitative 3D analysis of bone in hip osteoarthritis using clinical computed tomography
}

\author{
Tom D. Turmezei ${ }^{1,2} \cdot$ Graham M. Treece $^{1}$ Andrew H. Gee ${ }^{1}$ Anastasia F. Fotiadou $^{3}$. \\ Kenneth E. S. Poole ${ }^{4}$
}

Received: 17 March 2015 /Revised: 11 September 2015 / Accepted: 24 September 2015 /Published online: 7 October 2015

(C) The Author(s) 2015. This article is published with open access at Springerlink.com

\begin{abstract}
Objective To assess the relationship between proximal femoral cortical bone thickness and radiological hip osteoarthritis using quantitative $3 \mathrm{D}$ analysis of clinical computed tomography (CT) data.

Methods Image analysis was performed on clinical CT imaging data from 203 female volunteers with a technique called cortical bone mapping (CBM). Colour thickness maps were created for each proximal femur. Statistical parametric mapping was performed to identify statistically significant differences in cortical bone thickness that corresponded with the severity of radiological hip osteoarthritis. Kellgren and Lawrence (K\&L) grade, minimum joint space width (JSW) and a novel CT-based osteophyte score were also blindly assessed from the CT data.

Results For each increase in K\&L grade, cortical thickness increased by up to $25 \%$ in distinct areas of the superolateral
\end{abstract}

Electronic supplementary material The online version of this article (doi:10.1007/s00330-015-4048-x) contains supplementary material, which is available to authorized users.

Tom D. Turmezei

tdt21@cam.ac.uk

1 Department of Engineering, University of Cambridge, Trumpington Street, Cambridge CB2 1PZ, UK

2 Department of Radiology, Addenbrooke's Hospital, Cambridge Biomedical Campus, Hills Road, Box 218, Cambridge CB2 0QQ, UK

3 Department of Radiology, Royal National Orthopaedic Hospital, Brockley Hill, Stanmore, Middlesex HA7 4LP, UK

4 Department of Medicine, Addenbrooke's Hospital, Cambridge Biomedical Campus, Hills Road, Box 157, Cambridge CB2 0QQ, UK femoral head-neck junction and superior subchondral bone plate. For increasing severity of CT osteophytes, the increase in cortical thickness was more circumferential, involving a wider portion of the head-neck junction, with up to a $7 \%$ increase in cortical thickness per increment in score. Results were not significant for minimum JSW.

Conclusions These findings indicate that quantitative 3D analysis of the proximal femur can identify changes in cortical bone thickness relevant to structural hip osteoarthritis.

Key Points

- CT is being increasingly used to assess bony involvement in osteoarthritis

- CBM provides accurate and reliable quantitative analysis of cortical bone thickness

- Cortical bone is thicker at the superior femoral head-neck with worse osteoarthritis

- Regions of increased thickness co-locate with impingement and osteophyte formation

- Quantitative 3D bone analysis could enable clinical disease prediction and therapy development

Keywords Osteoarthritis · Hip joint · Bone - Helical CT . Imaging, three-dimensional
Abbreviations
CBM Cortical bone mapping
CT Computed tomography
DRR Digitally reconstructed radiographs
JSW Joint space width
K\&L Kellgren and Lawrence
MRI Magnetic resonance imaging
ROI Region of interest
SPM Statistical parametric mapping 


\section{Introduction}

Hip osteoarthritis is an enormous health burden estimated to affect one in four individuals in the USA during their lifetime [1]. Frustratingly there are no disease-modifying therapies currently approved for the treatment of any form of osteoarthritis, with substantial breakthroughs in therapy development hampered by an ongoing need for reliable biomarkers $[2,3]$.

Clinical and epidemiological studies continue to define disease with radiographic Kellgren and Lawrence $(K \& L)$ grading [4], but this system has been shown to suffer from variable interpretation [5]. The current recommended definition of disease progression for clinical trials is change in radiographic minimum joint space width (JSW) [6]. However, relevant progression needs to be defined within each study, which can complicate comparisons between different populations, while techniques of JSW measurement have been reported to have poor reproducibility [7, 8]. Radiographic assessment is also limited by $2 \mathrm{D}$ interpretation of a 3D structure.

Magnetic resonance imaging (MRI) stands at the fore of imaging in osteoarthritis research, but despite promise from a variety of structural and biochemical techniques, quantitative MRI has not yet delivered a substantiated osteoarthritis biomarker. However, clinical computed tomography (CT) has gained momentum in imaging of osteoarthritis in recent years [8-10]. This is because bone has become increasingly apparent as a key player alongside cartilage and synovium. It is also apt as a modality given the proposed structural mechanisms of femoro-acetabular impingement [11]. Although not yet approved for clinical use in humans, there have been encouraging reports on the efficacy of a bonerelated agent, strontium ranelate, from one clinical trial [12], providing motivation to monitor mineralised tissues. Quantitative CT of bone is further supported by recent opinion that careful alignment of therapeutic action, tissue response and imaging should yield the greatest chance of detecting longitudinal change with the required sensitivity [13].

In line with these strategies, our primary aim was to assess the relationship between cortical bone thickness and radiological hip osteoarthritis with a view to developing a new method for disease quantification. We propose this through the application of a recognised 3D image analysis technique called cortical bone mapping (CBM) [14-16]. Our hypothesis was that CBM would be able to identify regions of cortical bone thickening around the proximal femur associated with worse radiological osteoarthritis, predicting this would be in the distribution of recognised peri-articular bone-related phenomena such as osteophytes and subchondral sclerosis.

\section{Material and methods}

\section{Participants}

Participants for this observational study were taken from a cohort of 204 female control volunteers involved in existing Cambridge trials investigating hip fracture risk (57 from FEMCO: LREC 07-H0305-61; 38 from MRC-Hip fx and 100 from MRC-Ageing: LREC 06/Q0108/180; 9 from MRC-Stroke: LREC 01/245). The local research ethics committee approved each of these studies, with each participant giving informed consent for the analysis of their CT data for the investigation of hip disease. All participants were free of hip fracture, osteomyelitis, bone malignancy, unilateral metabolic bone disease, terminal illness and prior treatment with teriparatide or strontium ranelate. All examinations were reported by a consultant radiologist as part of the routine clinical care of patients. One of the 204 examinations was unavailable for analysis on account of data corruption and so imaging was used from 203 individuals. The mean $[ \pm$ standard deviation (SD)] age of this final group was $65 \pm 18$ years; mean weight was $70.1 \pm 14.4 \mathrm{~kg}$; mean height was $1.62 \pm 0.07 \mathrm{~m}$; mean body mass index (BMI) was $26.9 \pm 5.4 \mathrm{~kg} / \mathrm{m}^{2}$. No height data was available for one individual and so she was given the sample mean value.

\section{Imaging acquisition}

Imaging of the hips was acquired helically in the supine position on five different clinical whole-body multi-detector CT machines (Siemens SOMATOM Sensation 16, Sensation 64, Definition Flash, Definition AS+, and a GE Medical Systems Discovery 690). The acquisition protocol involved imaging inclusively from the superior margin of the acetabuli to the lesser trochanters. All acquisitions were processed with a standard smooth-edge body kernel and reconstructed with axial slice separation ranging from 0.75 to $1.5 \mathrm{~mm}$, equivalent to slice thickness in all cases. In-plane pixel spacing varied from 0.59 to $0.78 \mathrm{~mm}$, with field of view consistently 512 by 512 pixels. Peak kV was routinely $120 \mathrm{kV}$. When available from the fully anonymised metadata, recorded exposure ranged from 67-274 mAs, varying due to routine use of dose limiting.

\section{Radiological measures of hip osteoarthritis}

Since there were no clinical measures of disease available in this cohort, we used radiological scores to grade disease severity. Digitally reconstructed radiographs (DRRs) were created using an anatomically standardised coronal mean intensity projection with a window level of 200 Hounsfield units (HU) and a width of $700 \mathrm{HU}$. DRRs were reviewed by a consultant radiologist (AF) for $\mathrm{K} \& \mathrm{~L}$ grading (0 to 4 ) 
[4] and minimum JSW, recorded to the nearest $0.1 \mathrm{~mm}$ with electronic callipers. Multi-planar reformats were reviewed by another consultant radiologist (TT) for osteophyte load, a reviewer-generated cumulative score of osteophytes graded from 0 to 3 in 42 separate sectors from around the proximal femur [10]. The maximum score in this cohort was 47 , and so this was the upper limit used in our analysis. Full details of DRR and scoring methodologies (including reliability) have been previously published $[8,10]$.

We investigated the effect of these radiological measures on cortical thickness in the proximal femur, with the assumption of a linear relationship with disease for each. Frequency histograms were created for each radiological score using MATLAB 2013a (C 1984-2013, The MathsWork, Inc.) to demonstrate the spread of disease severity (Fig. 1). The assumption of linearity was validated by scatter plots and calculation of Kendall's tau correlation coefficient using StatPlus ${ }^{\circledR}$ :mac 2009 (AnalystSoft, StatPlus:mac - statistical analysis program for Mac OS. Version 2009; http://www.analystsoft.com/en/). Each covariate showed a linear relationship and significant correlation with the other (Electronic Supplementary Material 1). When both femurs were available, the side with the worse disease score was selected for analysis in order to minimise the risk of including only those with no or minimal disease. If disease scores were equal, then a side was chosen randomly for inclusion. Repeating analyses with different randomisation did not affect the pattern or significance of our results.

\section{Cortical thickness measurement}

Cortical thickness measurement was performed using CBM, implemented by a freely available in-house program called Stradwin (http://mi.eng.cam.ac.uk/ rwp/stradwin/). The main strength of this technique is its ability to accurately estimate cortical bone thickness below the pixel spacing of the imaging system, irrespective of partial volume effect. It has been validated for clinical CT against high resolution quantitative $\mathrm{CT}$ data acquired at 82 microns per pixel, and applied in clinical CT data sets up to a slice separation of $3 \mathrm{~mm}[14,17,18]$. Following semi-automatic segmentation and proximal femur mesh generation, cortical thickness was measured at each of $\sim 6,000$-mesh vertices at a normal to the underlying cortical surface. Creation of a single 3D thickness map takes, on average, 15 minutes; the majority of this time is spent on semi-automatic segmentation, which varies according to noise in the data, while automatic thickness analysis usually takes under 1 minute on a standard desktop computer. Measurement error $( \pm \mathrm{SD})$ at any given point has been previously reported as $0.12 \pm 0.39 \mathrm{~mm}$, well below the pixel spacing size in this study and otherwise in daily clinical practice [18]. Precision has also been assessed in repeat analysis of 19 individuals re-imaged with $\mathrm{CT}$ and re-analysed after an average of 61 days (SD 22 days, range 34 to 107 days) giving a measurement precision of $5 \%$ of the mean at individual points, dropping to $1 \%$ of the mean when measurements were averaged over a defined region of interest (ROI) [19].

In order to account for differences in hip morphology, each proximal femur was spatially re-aligned with a canonical right femur using B-spline free-form deformation as calculated by an iterative closest-point registration algorithm [20]. This was performed using freely available in-house software called WxRegSurf (http://mi.eng.cam.ac.uk/ ahg/wxRegSurf/). Spatially normalised cortical thickness maps were then smoothed with a 10-mm full width at half maximum (FWHM) filter, required for statistical parametric mapping (SPM). After registration of each bone to the canonical femur, SPM was performed to determine regions of significantly different cortical thickness for changes in radiological disease score.

\section{Statistical parametric mapping (SPM)}

SPM was initially developed for applications in neuroimaging to allow regionally located statistical inferences to be made from the comparison of data at multiple points in space. It uses a general linear model to account for variability in measurement data (here, cortical thickness) in terms of experimental a

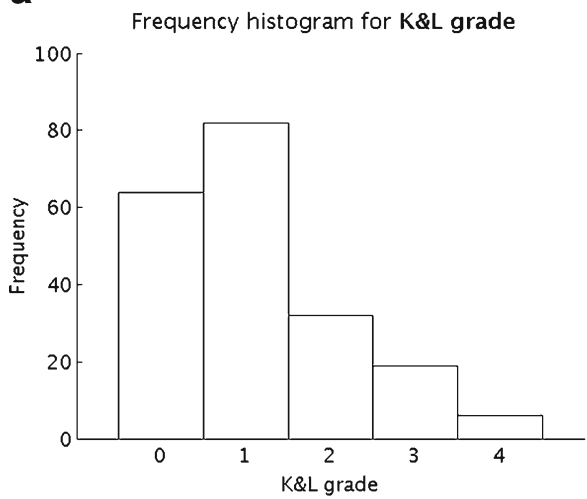

b

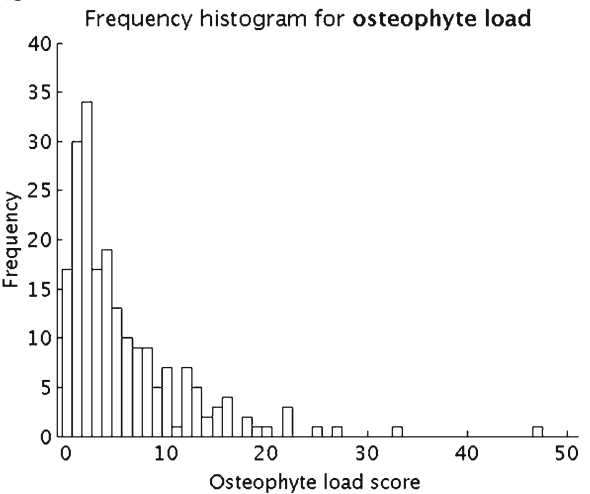

C

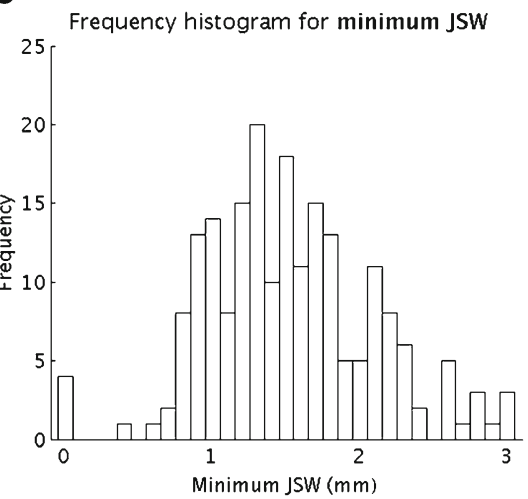

Fig. 1 Frequency histograms for each of the radiological scores. a Kellgren and Lawrence (K\&L) grade; b osteophyte load; and $\mathbf{c}$ minimum JSW at $0.1-\mathrm{mm}$ gradations 
and confounding effects. Effects in our general linear model were radiological disease score (experimental), age and weight (confounding, as revealed by preliminary analysis). Standard two-tail $\mathrm{F}$ testing across the cohort gave $p$-values at each measurement location that were uncorrected for multiple comparisons. As required for SPM, random field theory delivered $p$-values corrected for these multiple comparisons to control for false positive results (type I errors) [21]. SPM was performed using the SurfStat package [22] in MATLAB 2013a (C 1984-2013, The MathsWork, Inc.). A mean thickness map was also produced for the whole cohort (Fig. 2), allowing change in cortical thickness to be represented as a percentage of the mean. Therefore, three sets of results were calculated for each disease score:

(a) Absolute cortical thickening (in $\mathrm{mm}$ ) for each unit worsening in radiological measure;

(b) Relative cortical thickening (as a percentage of the mean value) for each unit worsening in radiological measure; and

(c) Subsequent regions of significant differences in cortical bone thickness across the cohort.

Significant regions of cortical bone thickness difference are "clusters" that meet statistical significance when tested after random field theory correction. For the purposes of visualising results effectively, all non-significant regions were indicated by washed out colour over the relative thickness maps (Figs. 3, 4, and 5). Absolute cortical thickness maps are included in the Electronic Supplementary Material (2 to 4) for reference against these relative thickness maps. An overview of the CBM pathway from data acquisition to results is presented as a flowchart in Fig. 6.

\section{Results}

Cortical bone mapping results for three radiological measures of disease (K\&L grade, osteophyte load and minimum JSW) are shown as a colour map on a canonical model in Figs. 3, 4, and 5, with non-significant regions according to SPM indicated by faded colours. Therefore, significant regions of cortical bone thickness difference with worsening disease score $(p<0.05)$ are represented in full colour in contrast to faded regions of non-significance.

\section{Kellgren and Lawrence $(K \& L)$ grade}

There was up to $25 \%$ significantly thicker cortical bone for each unit increase in K\&L grade at the superolateral and anterior femoral head-neck junction, with extension into the superior subchondral bone plate (Fig. 3). The map for absolute differences in cortical thickness is presented in Electronic Supplementary Material 2, showing that $25 \%$ was equivalent to a $0.1 \mathrm{~mm}$ increase per each unit increase in grade (0 to 4$)$.

\section{Osteophyte load}

There was up to $7 \%$ significantly thicker cortical bone for each unit increase in osteophyte load score circumferentially around the head-neck junction, extending medially across the superior subchondral bone plate to the fovea, and laterally into the posterior and medial femoral neck (Fig. 4). The map for absolute differences in cortical thickness is presented in Electronic Supplementary Material 3, showing that $7 \%$ was equivalent to a $0.02 \mathrm{~mm}$ increase per each unit increase in osteophyte load (0 to 47 in this cohort).

\section{Minimum joint space width (JSW)}

There was no significant difference in cortical bone thickness for each mm decrease in minimum JSW (Fig. 5), although cortical thickness at the superolateral head-neck junction still increased by up to $20 \%$ for each $\mathrm{mm}$ decrease in minimum JSW. The map for absolute differences in cortical thickness for minimum JSW is presented in Electronic Supplementary Material 4, showing that $20 \%$ was equivalent to a $0.08 \mathrm{~mm}$ increase per each $\mathrm{mm}$ decrease in minimum JSW.

\section{Discussion}

We have applied a quantitative 3D image analysis technique to clinical CT imaging data to identify significant cortical bone

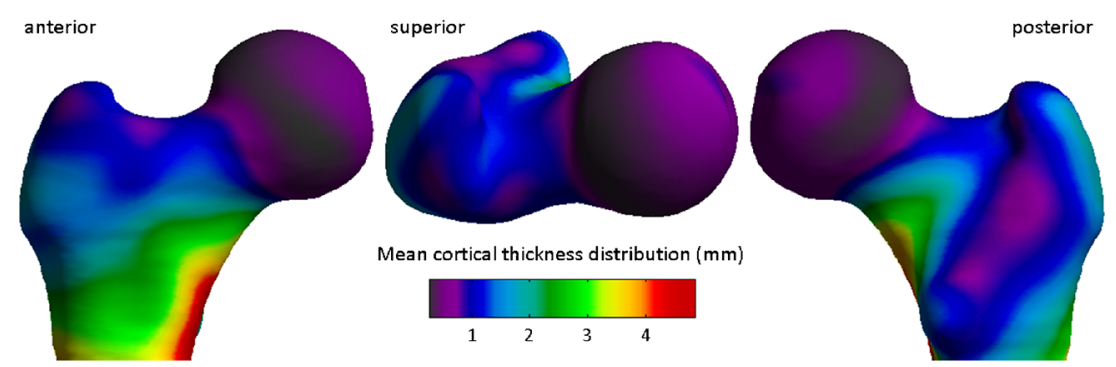

Fig. 2 Mean cortical thickness of the proximal femur. Mean cortical thickness map of the proximal femur from the 203 individuals in the study cohort, presented as a colour wash on a canonical model of the right femur viewed from three perspectives: (left to right) anterior, superior and posterior 


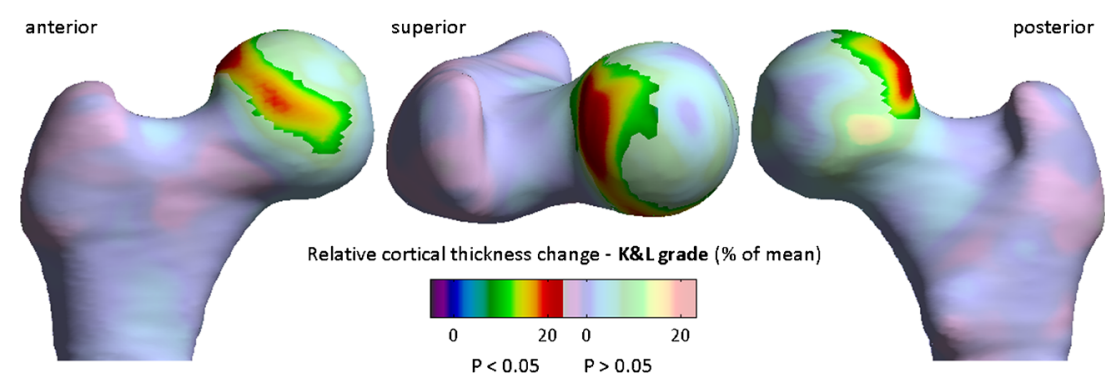

Fig. 3 Cortical bone mapping (CBM) results for Kellgren and Lawrence $(\mathrm{K} \& \mathrm{~L})$ grade. $\mathrm{CBM}$ results for $\mathrm{K} \& \mathrm{~L}$ grade presented as a colour wash on the canonical right proximal femur. Significant regions of cortical bone thickness difference for each unit increase in $K \& L$ grade $(0$ to 4$)$ are represented by the colour map $(p<0.05)$, with non-significant regions indicated by faded colours $(p>0.05)$

same study also localised stress maxima within subchondral bone, supporting the notion that superolateral impingement is influencing structural change here. It is, therefore, possible that we are detecting a subclinical response in cortical bone to mechanical effects of impingement at the superolateral head-neck junction. CBM may also be detecting a cortical manifestation of osteophyte growth. Figure 7 shows that this may not necessarily be an osteophyte component outside the cortex, since our algorithm selects the innermost of two layers as the true cortex for analysis. Importantly, peri-articular cortical bone and subchondral bone plate can appear thickened as separate from osteophytes, or even in their absence, as review of the underlying imaging data reveals (Fig. 7).

Before considering the next phase of validation for assessment of osteoarthritis with CBM, it is important to evaluate the implications of using clinical CT for this purpose. CT is important for imaging joints and bone because it excels at depicting mineralised structures in contrast to surrounding soft tissue. In addition to providing a framework for finite element studies [24-26], CT has also shown strength in representing bone shape and early bone-related changes of disease at the hip [27, 28]. The promotion of CT for imaging large joint osteoarthritis should, therefore, be balanced against its weaknesses and the inherent values of other modalities such as MRI and radiography. This is of particular importance given the exposure to ionising radiation that it entails along with the proximity of radiation-sensitive tissues in the pelvis (which is, therefore, relatively less dose at more distal joints such as the knee). However the continual development

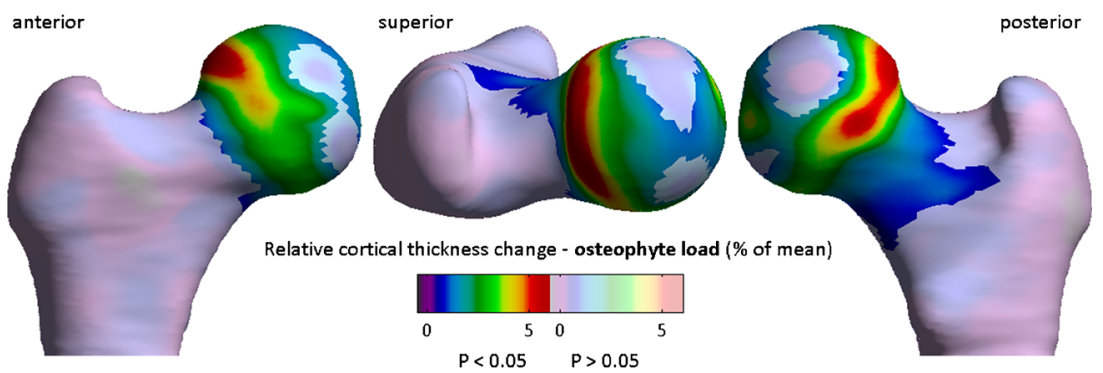

Fig. 4 Cortical bone mapping (CBM) results for osteophyte load. CBM results for osteophyte load presented as a colour wash on the canonical right proximal femur. Significant regions of cortical bone thickness

difference for each unit increase in osteophyte load score are represented by the colour map $(p<0.05)$, with non-significant regions indicated by faded colours $(p>0.05)$ 


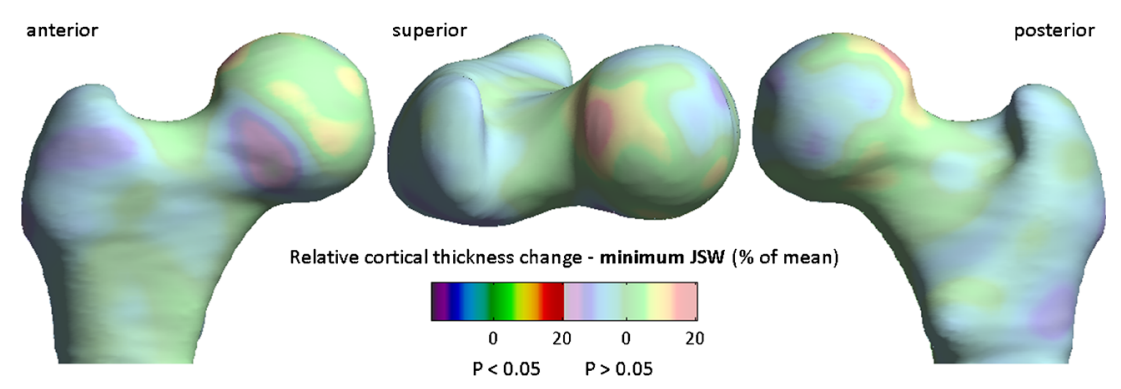

Fig. 5 Cortical bone mapping (CBM) results for minimum joint space width (JSW). CBM results for minimum JSW presented as a colour wash on the canonical right proximal femur. All colours were faded for this variable, meaning that there were no regions of significance $(p>0.05)$

of dose reduction strategies should encourage consideration for CT in clinical trials $[29,30]$.

We recognise that this study has limitations. Our assessment of hip osteoarthritis severity was based on imaging

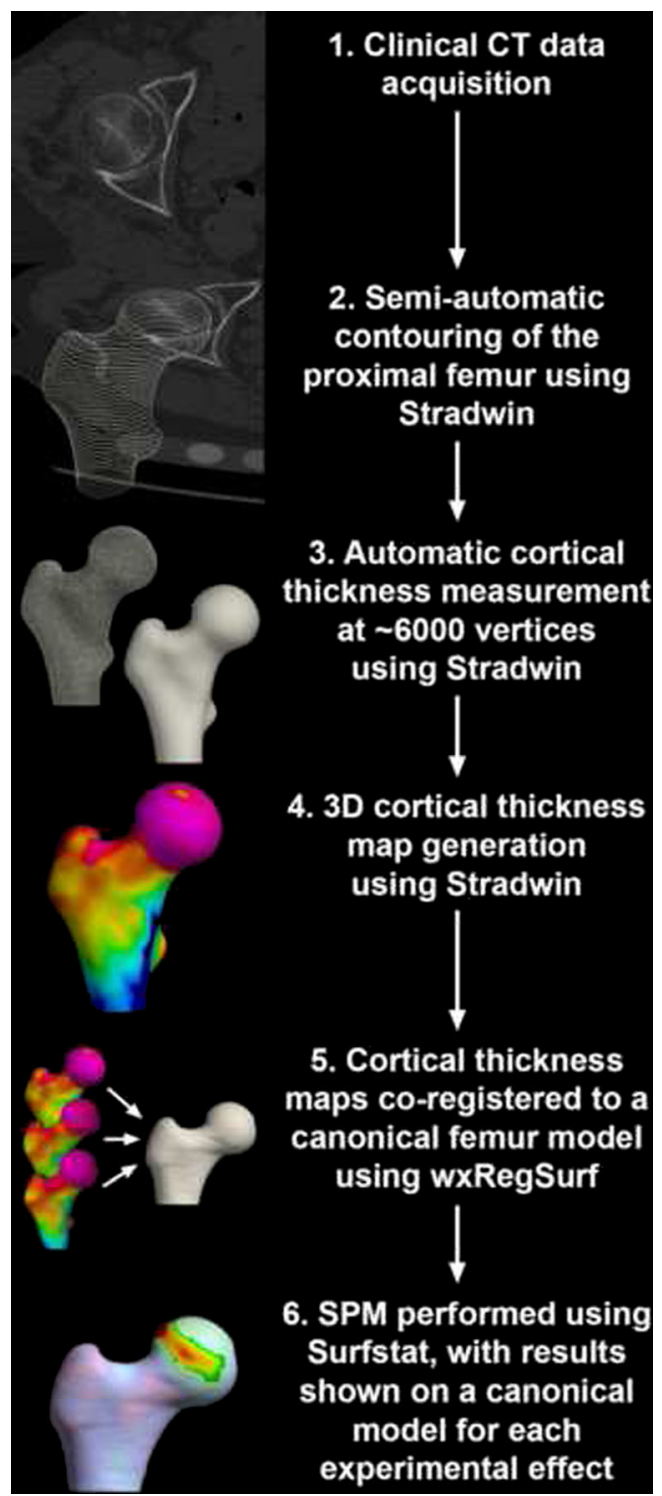

Fig. 6 Cortical bone mapping (CBM) flowchart. Each stage of the CBM pathway is represented to summarise the study methodology. SPM = statistical parametric mapping features alone, which do not necessarily correlate with clinical osteoarthritis. Thus, the next step would be to test the hypothesis that increased peri-articular cortical bone thickness can predict worse disease according to clinical outcome measures. We also recognise that individuals from this cohort were preselected on the basis of volunteering for studies in which the aim was to establish hip fracture risk, and so there will be according selection bias. Finally, subchondral bone sclerosis is a recognised radiological feature used in assessment with $\mathrm{K} \& \mathrm{~L}$ grading, meaning that there will be some dependence of the output of our cortical thickness analysis on $\mathrm{K} \& \mathrm{~L}$ grade, since DRRs were created from the same CT data. Nonetheless, we believe that CBM should now be tested to show whether quantitative 3D measurement of cortical bone with clinical CT can predict clinical disease with the necessary levels of sensitivity and precision [31]. The role of CBM in the prediction of osteoarthritis at other large joints, such as the knee, should also be evaluated.

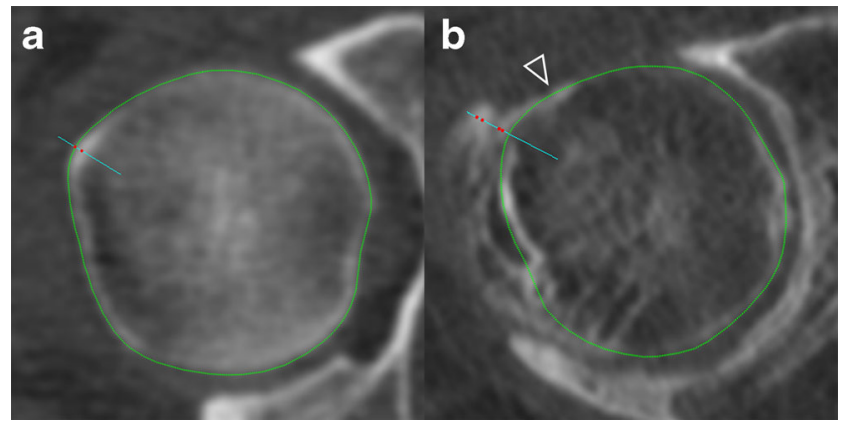

Fig. 7 Cortical thickness at the superolateral femoral head-neck junction. Axial CT images of the right hip in two individuals from the cohort viewed in Stradwin, the software used to perform CBM. Dotted green lines represent the approximate contour for the femoral isosurface. Cyan lines represent the path of thickness measurement. Red dots represent the estimated limits of cortical bone, neither of which need be coincident with the original green contour. Individual (a) had a Kellgren and Lawrence (K\&L) grade of 1, an osteophyte load of 4 (out of a maximum of 126), and a minimum JSW of $2.0 \mathrm{~mm}$. Note that the measurement line is at a site with apparently greater cortical thickness than surrounding bone yet in the absence of an osteophyte. Individual (b) had a K\&L grade of 3, an osteophyte load of 44 (out of 126), and a minimum JSW of $1.2 \mathrm{~mm}$. Note that the measurement line in (b) has picked up two layers of bone, the underlying cortex and the osteophyte outside it. Note that there is also apparent increased cortical bone thickness in the anterior femoral head anteromedially and separate to the osteophyte (arrowhead) 


\section{Conclusion}

CBM applied to the proximal femur in clinical CT imaging data identified significant structural changes in peri-articular cortical bone thickness that were associated with worse radiological hip osteoarthritis, particularly at the superolateral head-neck junction. These findings are best explained by the manifestation of osteophytes and subchondral sclerosis in this region, which may be either in response to local biomechanical stresses or the biochemical environment as the joint fails. CBM could, therefore, have an impact on both osteoarthritis research and subsequent clinical practice, especially with potential for application in disease monitoring and the development of structurally modifying therapies.

Acknowledgements The scientific guarantor of this publication is Dr. Kenneth Poole, University Lecturer in Metabolic Bone Disease, University of Cambridge. The authors of this manuscript declare relationships with the following companies: Dr Kenneth Poole and Dr Tom Turmezei have received honoraria from AMGEN Inc. through Cambridge Enterprise. Dr Poole is also a member of the AMGEN advisory board. Dr Andrew Gee and Dr Graham Treece have received grants from AMGEN Inc. and Eli Lilly and Company for research unrelated to this study. This study has received funding by Arthritis Research UK (Research Progression award, RG66087), the Evelyn Trust (Clinical Training Fellowship award, RG65411) and the Cambridge National Institute of Health Research Biomedical Research Centre (RG64246).

Two of the authors (Dr Graham Treece and Dr Andrew Gee) have significant statistical expertise. Institutional review board approval was obtained. Written informed consent was obtained from all subjects (patients) in this study.

Some study subjects or cohorts have been previously reported in:

Turmezei TD, Lomas DJ, Fotiadou A, Hopper MA, Poole KES. A new CT grading system for hip osteoarthritis. Osteoarthritis Cartilage 2014 Oct;22(10):1360-6

Turmezei TD, Lomas DJ, Hopper MA, Poole KES. Severity mapping of the proximal femur: a new method for assessing hip osteoarthritis with computed tomography. Osteoarthritis Cartilage 2014 Oct;22(10):1488-98

These two papers used the same cohorts for the development of CT grading of hip osteoarthritis as an entirely separate research methodology.

Methodology: retrospective, observational, performed at one institution.

Open Access This article is distributed under the terms of the Creative Commons Attribution-NonCommercial 4.0 International License (http:// creativecommons.org/licenses/by-nc/4.0/), which permits any noncommercial use, distribution, and reproduction in any medium, provided you give appropriate credit to the original author(s) and the source, provide a link to the Creative Commons license, and indicate if changes were made.

\section{References}

1. Murphy LB, Helmick CG, Schwartz TA et al (2010) One in four people may develop symptomatic hip osteoarthritis in his or her lifetime. Osteoarthr Cartil 18:1372-1379

2. Williams FM, Spector TD (2008) Biomarkers in osteoarthritis. Arthritis Res Ther 10:101
3. Hellio Le Graverand-Gastineau MP (2009) OA clinical trials: current targets and trials for OA. Choosing molecular targets: what have we learned and where we are headed? Osteoarthr Cartil 17: 1393-1401

4. Kellgren JH, Lawrence JS (1957) Radiological assessment of osteo-arthrosis. Ann Rheum Dis 16:494-502

5. Schiphof D, Boers M, Bierma-Zeinstra SM (2008) Differences in descriptions of Kellgren and Lawrence grades of knee osteoarthritis. Ann Rheum Dis 67:1034-1036

6. Ornetti P, Brandt K, Hellio-Le Graverand MP et al (2009) OARSIOMERACT definition of relevant radiological progression in hip/ knee osteoarthritis. Osteoarthr Cartil 17:856-863

7. Gossec L, Jordan JM, Lam MA et al (2009) Comparative evaluation of three semi-quantitative radiographic grading techniques for hip osteoarthritis in terms of validity and reproducibility in 1404 radiographs: report of the OARSI-OMERACT Task Force. Osteoarthr Cartil 17:182-187

8. Turmezei TD, Fotiadou A, Lomas DJ, Hopper MA, Poole KE (2014) A new CT grading system for hip osteoarthritis. Osteoarthr Cartil 22:1360-1366

9. Bousson V, Lowitz T, Laouisset L, Engelke K, Laredo JD (2012) CT imaging for the investigation of subchondral bone in knee osteoarthritis. Osteoporos Int 23:S861-S865

10. Turmezei TD, Lomas DJ, Hopper MA, Poole KE (2014) Severity mapping of the proximal femur: a new method for assessing hip osteoarthritis with computed tomography. Osteoarthr Cartil 22: $1488-1498$

11. Agricola R, Waarsing JH, Arden NK et al (2013) Cam impingement of the hip-a risk factor for hip osteoarthritis. Nat Rev Rheumatol 9: 630-634

12. Reginster JY, Badurski J, Bellamy N et al (2013) Efficacy and safety of strontium ranelate in the treatment of knee osteoarthritis: results of a double-blind, randomised placebo-controlled trial. Ann Rheum Dis 72:179-186

13. Eckstein F, Guermazi A, Gold G et al (2014) Imaging of cartilage and bone: promises and pitfalls in clinical trials of osteoarthritis. Osteoarthr Cartil 22:1516-1532

14. Treece GM, Gee AH (2015) Independent measurement of femoral cortical thickness and cortical bone density using clinical CT. Med Image Anal 20:249-264

15. Poole KE, Treece GM, Mayhew PM et al (2012) Cortical thickness mapping to identify focal osteoporosis in patients with hip fracture. PLoS One 7, e38466

16. Poole KE, Treece GM, Gee AH et al (2014) Denosumab rapidly increases cortical bone in key locations of the femur: a 3D bone mapping study in women with osteoporosis. J Bone Miner Res 30: 46-53

17. Treece GM, Gee AH, Mayhew PM, Poole KE (2010) High resolution cortical bone thickness measurement from clinical CT data. Med Image Anal 14:276-290

18. Treece GM, Poole KE, Gee AH (2012) Imaging the femoral cortex: thickness, density and mass from clinical CT. Med Image Anal 16: 952-965

19. Treece GM, Gee AH, Tonkin C, et al. (2015) Predicting hip fracture type with cortical bone mapping (CBM) in the Osteoporotic Fractures in Men (MrOS) study (Technical Report). Cambridge University Engineering Department Cambridge UK. Available via http://mi.eng.cam.ac.uk/reports/abstracts/biomed/ treece_tr695.html. Accessed 1 Mar 2015

20. Rueckert D, Frangi AF, Schnabel JA (2003) Automatic construction of 3-D statistical deformation models of the brain using nonrigid registration. IEEE Trans Med Imaging 22:1014-1025

21. Friston KJ, Holmes AP, Worsley KJ, Poline JP, Frith CD, Frackowiak RSJ (1994) Statistical parametric maps in functional imaging: a general linear approach. Hum Brain Mapp 2:189-210 
22. Worsley K, Taylor J, Carbonell F, et al. A Matlab toolbox for the statistical analysis of univariate and multivariate surface and volumetric data using linear mixed effects models and random field theory. NeuroImage Organisation for Human Brain Mapping 2009 Annual Meeting 47:S102

23. Hayami T, Pickarski M, Wesolowski GA et al (2004) The role of subchondral bone remodeling in osteoarthritis: reduction of cartilage degeneration and prevention of osteophyte formation by alendronate in the rat anterior cruciate ligament transection model. Arthritis Rheum 50:1193-1206

24. Anderson AE, Ellis BJ, Maas SA, Peters CL, Weiss JA (2008) Validation of finite element predictions of cartilage contact pressure in the human hip joint. J Biomech Eng 130:051008

25. Ng KC, Rouhi G, Lamontagne M, Beaulé PE (2012) Finite element analysis examining the effects of cam FAI on hip joint mechanical loading using subject-specific geometries during standing and maximum squat. HSS J 8:206-212

26. McErlain DD, Milner JS, Ivanov TG, Jencikova-Celerin L, Pollmann SI, Holdsworth DW (2011) Subchondral cysts create increased intra-osseous stress in early knee OA: a finite element analysis using simulated lesions. Bone 48:639-646

27. Harris MD, Datar M, Whitaker RT, Jurrus ER, Peters CL, Anderson AE (2013) Statistical shape modeling of cam femoroacetabular impingement. J Orthop Res 31:1620-1626

28. Siebelt M, Agricola R, Weinans H, Kim YJ (2014) The role of imaging in early hip OA. Osteoarthr Cartil 22:1470-1480

29. Sodickson A (2013) Strategies for reducing radiation exposure from multidetector computed tomography in the acute care setting. Can Assoc Radiol J 64:119-129

30. Herts BR, Baker ME, Obuchowski N et al (2013) Dose reduction for abdominal and pelvic MDCT after change to graduated weightbased protocol for selecting quality reference tube current, peak kilovoltage, and slice collimation. AJR Am J Roentgenol 200: 1298-1303

31. Goodsaid FM, Mendrick DL (2010) Translational medicine and the value of biomarker qualification. Sci Transl Med 2, $47 \mathrm{ps} 44$ 